\title{
Research Article \\ Design of Sanda Action Reconstruction Model Based on 3D Images
}

\author{
Hongwei Zhao \\ Department of Physical Education, Guilin University of Electronic Technology, Guilin 541004, China \\ Correspondence should be addressed to Hongwei Zhao; zhaohongwei@guet.edu.cn
}

Received 20 October 2020; Revised 13 December 2020; Accepted 4 January 2021; Published 19 January 2021

Academic Editor: Hongju Cheng

Copyright (C) 2021 Hongwei Zhao. This is an open access article distributed under the Creative Commons Attribution License, which permits unrestricted use, distribution, and reproduction in any medium, provided the original work is properly cited.

\begin{abstract}
In order to improve the training level of Sanda movement, this article uses an image analysis method to reconstruct the detailed characteristics of the movement and apply them to the actual training process. Since the traditional wavelet reconstruction method is affected by the accuracy of the decomposition scale, this paper proposes an improved method of Sanda action based on 3D image reconstruction. First, the method relies on frame adjacent phase compensation and digital image stabilization techniques to perform digital frame operations on the image. Then, scanning and corner detection are used for image reconstruction, where adjacent phase compensation methods are used to match feature points and gray pixels. Image extraction is performed by extracting key feature points of the action 3D image, and a fast frame detection method is used to stabilize the image of the digital image, thereby improving the image quality of image reconstruction. The experimental results show that the method has good image output effect and has a high application value in Sanda guidance and optimization.
\end{abstract}

\section{Introduction}

Sanda is an important part of Chinese martial arts and is very different from daily sports [1]. The movement is characterized by various movements and different forms [2]. Participants beat and beat their opponents in accordance with certain rules [3]. On the basis of traditional Chinese martial arts, combining modern Western sports ideas, Sanda has gradually developed into modern martial arts [4]. Breakup is a competitive sport with strong confrontation. Therefore, in order to improve the level of scattering and obtain a pleasing effect, it is necessary to use the principles of computer and image processing technology to reconstruct the scattering behavior [5]. By combining the motion feature analysis method, the method of evaluating and exploring scattered movements reveals the principle of movements, thereby improving the standardization and rationality of movements [6].

With the prosperity of computer image processing, the definition of image quality and the ability of 3D presentation of Sanda action images are more demanding. The threedimensional image reconstruction is a combination of three-dimensional virtual reality technology and visual simulation technology [7]. High-definition image synthesis is accomplished, which exerts great significance on studying the $3 \mathrm{D}$ image reconstruction technology in promoting the optimization and upgrading of Sanda action and enhancing the technical quality [8]. Three-dimensional image reconstruction is based on image processing algorithms such as image perception, image information fusion, and image denoising filtering [9], making use of digital image stabilization and $3 \mathrm{D}$ reconstruction technology so as to achieve image upgrades and improve the reconstruction quality [10]. In this paper, a three-dimensional image reconstruction technique in accordance with frame adjacent phase compensation and digital image stabilization is proposed [11]. Digital frame scanning and corner detection are manufactured. The adjacent phase compensation method is adopted to match feature points and segment gray pixels [12]. By means of extracting the key feature points, the fast frame detection method is wielded to stabilize the image of the digital image so that the image quality of image reconstruction is ameliorated [13]. Finally, the performance test is carried out through 
the simulation experiment, which shows the superior performance of this method in improving the reconfiguration ability [14].

The specific contributions of this paper include the following:

(1) This article uses image analysis methods to reconstruct the detailed characteristics of the movement and apply them to the actual training process

(2) An improved Sanda action method based on 3D image reconstruction is proposed

(3) Image extraction was performed by extracting the key feature points of the action $3 \mathrm{D}$ image, and the fast frame detection method was used to stabilize the image of the digital image, thereby improving the image quality of image reconstruction

The rest of this paper is organized as follows. Section 2 discusses image collection and preprocessing of Sanda action, followed by realization of $3 \mathrm{D}$ image reconstruction as discussed in Section 3. Analysis of the simulation experiment is discussed in Section 4. Section 5 concludes the paper with a summary and future research directions.

\section{Image Collection and Preprocessing of Sanda Action}

2.1. Digital Frame Scanning and Corner Detection of Sanda Action. In order to reconstruct the three-dimensional image of Sanda action, it is initially necessary to scan the digital frame and detect the corner for the image picture, and the image scanning is performed by the frame detection method [15], combining feature sampling with laser scanning technology to achieve fast image processing. In the gravy region, the pixel size of the image is recorded as $M \times M$, and the image points are fused to each frame [16]. The image is processed by updating iterative $s(k)=[\theta(k), \Delta x(k), \Delta y(k)]$, and the pixel value of the $3 \mathrm{D}$ scanned image tends to be zero. The holographic information of the image is obtained by the variational principle. The normalized pixel values of image feature distribution in subpixel space are as below [17]:

$$
P(i, j)\left(i \in\left[0, \text { int }\left(\frac{W}{2}\right)-1\right], \quad j \in\left[0, \text { int }\left(\frac{H}{2}\right)-1\right]\right) \text {. }
$$

Within the range of known integer parallax, the subpixel parallax of Sanda action 3D image is obtained by the local maximum search for image pixel segmentation, as follows:

$$
\begin{aligned}
E_{\text {image }}(V i)= & -\mid I\left(x_{i}-1, y_{i}+1\right)+2 I\left(x_{i}, y_{i}+1\right) \\
& +I\left(x_{i}+1, y_{i}+1\right)-I\left(x_{i}-1, y_{i}-1\right) \\
& -2 I\left(x_{i}, y_{i}-1\right)-I\left(x_{i}+1, y_{i}-1\right) \mid \\
& +\mid I\left(x_{i}+1, y_{i}-1\right)+2 I\left(x_{i}+1, y_{i}\right) \\
& +I\left(x_{i}+1, y_{i}-1\right)-I\left(x_{i}-1, y_{i}-1\right) \\
& -2 I\left(x_{i}-1, y_{i}\right)-I\left(x_{i}-1, y_{i}+1\right) \mid .
\end{aligned}
$$

Consequently, the digital frame scanning and corner detection of three-dimensional Sanda action images are completed, which provides an accurate information input basis for $3 \mathrm{D}$ reconstruction [18].

2.2. Frame Adjacent Phase Compensation. Using motion feature point matching and gray pixel segmentation technology, the neighboring phase compensation of the frame output feature points is performed to extract the key feature points in the Sanda 3D image [19]. The frame distribution function of the Sanda action picture in view of gray projection is expressed as follows:

$$
\left\{\begin{array}{l}
t=\mu \cos \alpha-v \sin \alpha, \\
\omega=\mu \sin \alpha+v \cos \alpha .
\end{array}\right.
$$

The frequency hopping feature points of the Sanda action screen are matched by image stabilization and grayscale pixel segmentation. The contour length of the resulting 3D image reconstruction is as follows [20]:

$$
E=\theta E^{\mathrm{LBF}}+(1-\theta) E^{\mathrm{LGF}}+v L(\phi)+\mu P(\phi) .
$$

The window is $3 \times 3$, and the gray value of the pixel at $(i, j)$ is represented by $X_{i, j}$. The subpixel accuracy of adjacent phase compensation of the frame is calculated as follows [21]:

$$
L(\phi)=\int_{\Omega} \delta(\phi)|\nabla \phi| d x
$$

Given that $P(\phi)$ is a sparse regularized item, the subpixel matching method is adopted so as to compensate the image stabilization error of small edge pixels, and the parallax function is obtained as follows:

$$
P(\phi)=\int \frac{1}{2}(|\nabla \phi|-1)^{2} d x
$$

in which $E^{\mathrm{LBF}}$ is the parallax function and $E^{\mathrm{LGF}}$ is the local gradient energy term, aimed at the displacement invariance of image attribute features. The image is discretely processed to complete the feature point matching of the dynamic image of Sanda action. The grayscale pixel segmentation results of image reconstruction are obtained as

$$
\begin{aligned}
E^{\mathrm{LBF}}\left(\phi, f_{1}, f_{2}\right)= & \lambda_{1} \int\left[\int K_{\sigma}(x-y)\left|I-f_{1}(x)\right|^{2} H(\phi) d y\right] d x \\
& +\lambda_{2} \int\left[\int K_{\sigma}(x-y)\left|I-f_{2}(x)\right|^{2}(1-H(\phi)) d y\right] d x,
\end{aligned}
$$

$$
\begin{aligned}
E^{\mathrm{LGF}}\left(\phi, f_{1}^{G}, f_{2}^{G}\right)= & \lambda_{1} \int\left[\int K_{\sigma}(x-y)\left|I^{G}-f_{1}^{G}(x)\right|^{2} H(\phi) d y\right] d x \\
& +\lambda_{2} \int\left[\int K_{\sigma}(x-y)\left|I^{G}-f_{2}^{G}(x)\right|^{2}(1-H(\phi)) d y\right] d x,
\end{aligned}
$$




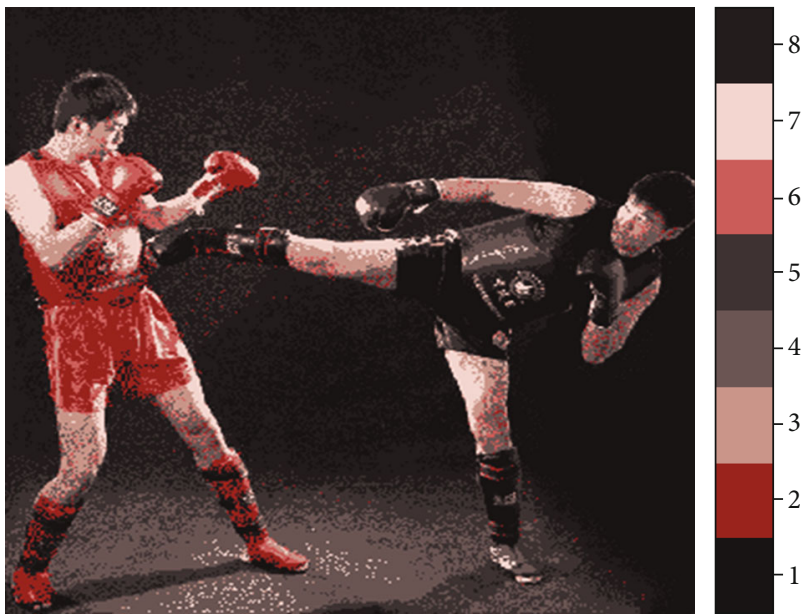

FIgURE 1: Original Sanda image acquisition result functions.

in which $I^{G}$ is the gradient mode and $f_{1}^{G}$ and $f_{2}^{G}$ represent the stereo matching support and gray window of 3D Sanda motioned image reconstruction, respectively. The original image acquisition and fractal dimension distribution are shown in Figures 1 and 2.

\section{Realization of 3D Image Reconstruction}

3.1. Digital Image Stabilization Processing. In view of the above-mentioned collection and preprocessing of Sanda moving images, this article uses the three-dimensional reconstruction of Sanda moving images for processing. The adjacent phase compensation and digital imaging technology are proposed to extract the basic technical feature points of the three-dimensional image. The feature equation is described as below:

$$
\begin{aligned}
\frac{\partial \phi}{\partial t}= & -\delta(\phi)\left[\theta\left(\lambda_{1} e_{1}^{\mathrm{LBF}}-\lambda_{2} e_{2}^{\mathrm{LBF}}\right)+(1-\theta)\left(\lambda_{1} e_{1}^{\mathrm{LGF}}-\lambda_{2} e_{2}^{\mathrm{LGF}}\right)\right] \\
& +v \delta(\phi) \operatorname{div}\left(\frac{\nabla \phi}{\nabla \phi}\right)+\mu\left(\nabla^{2} \phi-\operatorname{div}\left(\frac{\nabla \phi}{|\nabla \phi|}\right)\right),
\end{aligned}
$$

in which $e_{1}^{\mathrm{LBF}}, e_{2}^{\mathrm{LBF}}, e_{1}^{\mathrm{LGF}}$, and $e_{2}^{\mathrm{LGF}}$ are

$$
\begin{aligned}
& \left\{\begin{array}{l}
e_{1}^{\mathrm{LBF}}=\int_{\Omega} K_{\sigma}(y-x)\left|I(x)-f_{1}(y)\right|^{2} d y, \\
e_{2}^{\mathrm{LBF}}=\int_{\Omega} K_{\sigma}(y-x)\left|I(x)-f_{2}(y)\right|^{2} d y,
\end{array}\right. \\
& \left\{\begin{array}{l}
e_{1}^{\mathrm{LGF}}=\int_{\Omega} K_{\sigma}(y-x)\left|I^{G}(x)-f_{1}^{G}(y)\right|^{2} d y, \\
e_{2}^{\mathrm{LGF}}=\int_{\Omega} K_{\sigma}(y-x)\left|I^{G}(x)-f_{2}^{G}(y)\right|^{2} d y,
\end{array}\right.
\end{aligned}
$$

in which $H(\phi)$ is a Heaviside function, which represents the derivative of the data item of the $3 \mathrm{D}$ image reconstruction in Sanda movement as well as the derivative of the slip term.

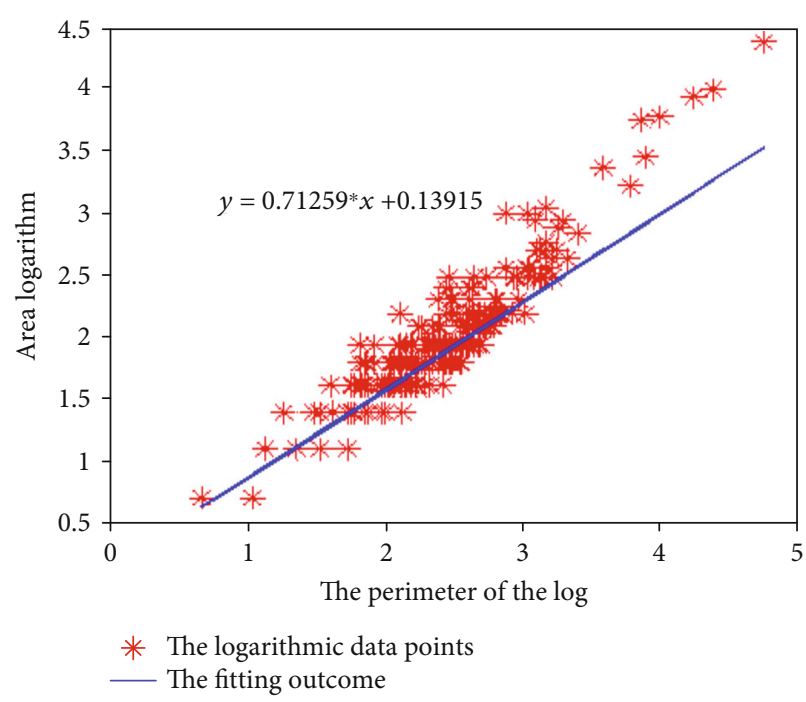

Figure 2: Original Sanda image fractal dimensions.

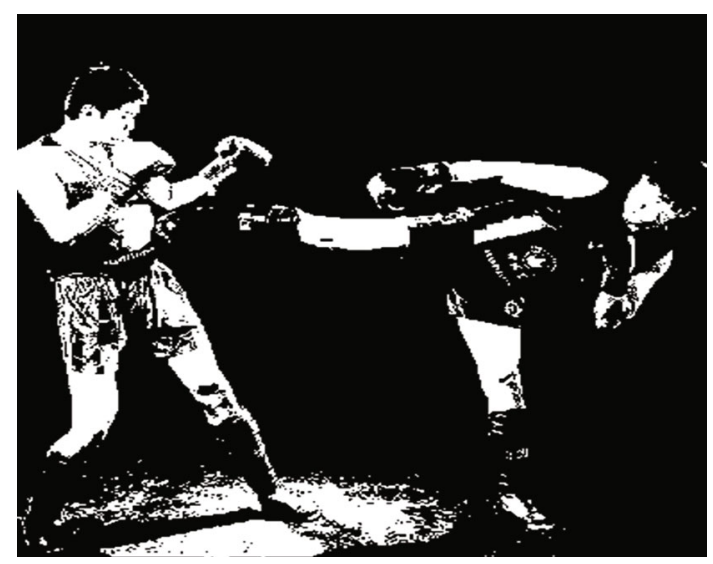

FIgURE 3: Reconstruction effect of Sanda movement and kinematic reconstruction.

$\delta(z)=-(d / d z) H(z)$ is the Dirac function, which is the dynamic point of the motion holographic imaging of the Sanda. The fast frame detection method is applied to carry out the digital image stabilization for the extracted feature points, and the pixels distributed in the image are regarded as templates. After the frame phase is compensated by the adjacent phase, the output picture and the parallax pixel after the image processing are obtained:

$$
\begin{aligned}
& H_{\varepsilon}(z)=\frac{1}{2}\left[1+\frac{2}{\pi} \arctan \left(\frac{Z}{\varepsilon}\right)\right], \\
& \delta_{\varepsilon}(z)=\frac{1}{\pi} \cdot \frac{\varepsilon}{\varepsilon^{2}+Z^{2}}, \quad z \in R .
\end{aligned}
$$

On account of the robustness of the SIFT algorithm, the SIFT algorithm is used for digital image stabilization, which is effectively in a position to increase the quality of image reconstruction. 


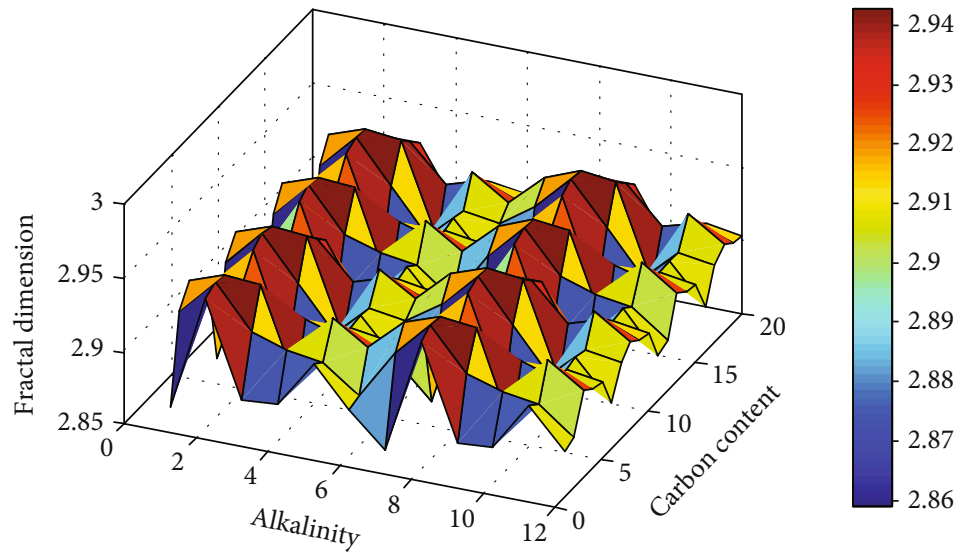

FigURE 4: Reconstruction graph fractal exponential of Sanda.

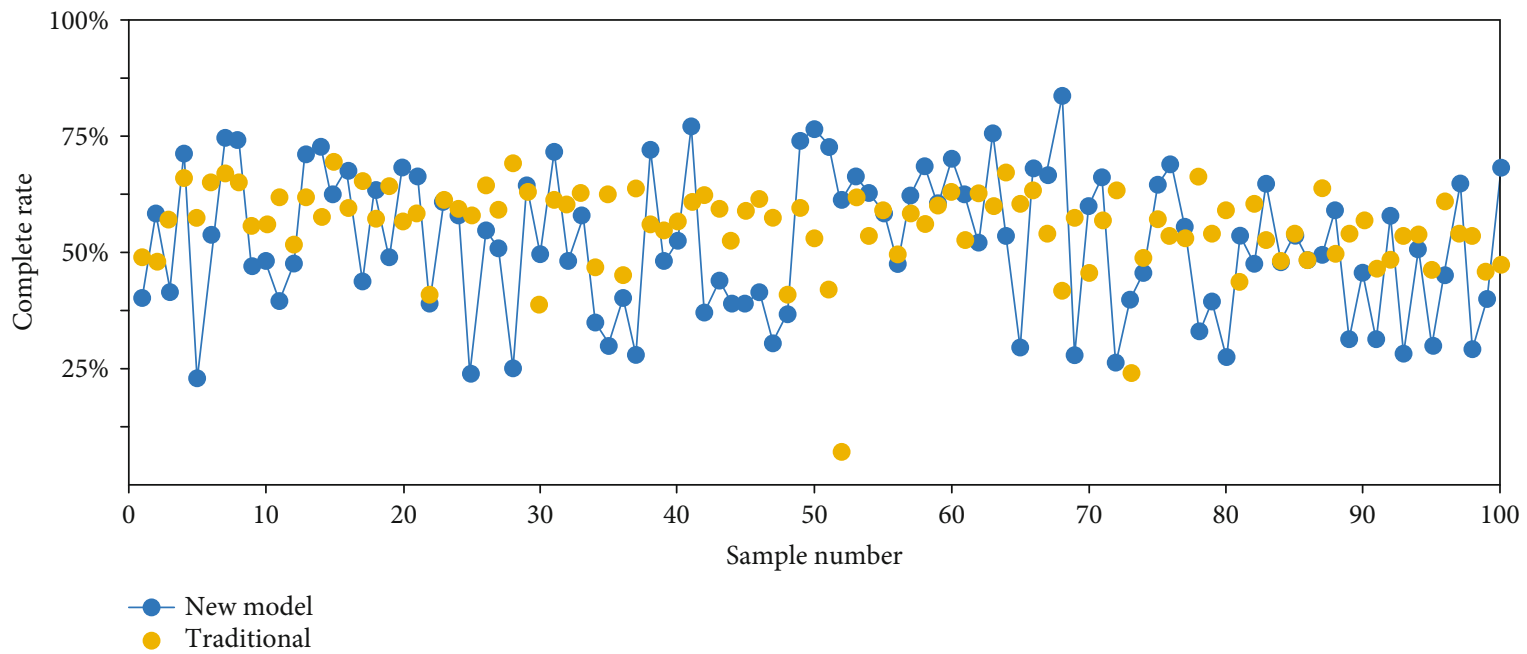

FIGURE 5: Comparable results of image reconstruction quality obtained by means of diverse methods.

3.1.1. 3D Image Reconstruction. According to image stabilization, the image-driven smoothing term is fixed, and the noise of the image boundary and parallax boundary is separated on the ray direction $\phi$ of image imaging, and the image-driven smoothing term is selected as the direct volume. The $3 \mathrm{D}$ reconstruction output of the image is realized; the output image expression is expressed as follows:

$$
\begin{aligned}
& \left\{\begin{array}{l}
f_{1}(x)=\frac{K_{\sigma}(x) *\left[H_{\varepsilon}(\phi(x)) I(x)\right]}{K_{\sigma}(x) * H_{\varepsilon}(\phi(x))}, \\
f_{1}(x)=\frac{K_{\sigma}(x) *\left[\left(1-H_{\varepsilon}(\phi(x))\right) I(x)\right]}{K_{\sigma}(x) *\left(1-H_{\varepsilon}(\phi(x))\right)},
\end{array}\right. \\
& \left\{\begin{array}{l}
f_{1}^{G}=\frac{K_{\sigma}(x) *\left[H_{\varepsilon}(\phi(x)) I^{G}(x)\right]}{K_{\sigma}(x) * H_{\varepsilon}(\phi(x))}, \\
f_{2}^{G}=\frac{K_{\sigma}(x) *\left[\left(1-H_{\varepsilon}(\phi(x))\right) I^{G}(x)\right]}{K_{\sigma}(x) *\left(1-H_{\varepsilon}(\phi(x))\right)} .
\end{array}\right.
\end{aligned}
$$

A binary gradient function $C=\{(x, y) \in \Omega: \phi(x, y)=0\}$ makes use of representing the gradient information of the image, and $N(i)$ is used to represent the immediately adjacent region of the pixel point $i$ of the Sanda action 3D image. The subpixel stereo output of the $3 \mathrm{D}$ image reconstruction is obtained as follows:

$$
\begin{aligned}
& \lim _{P \rightarrow+\infty} K_{B}^{P}(f)(x, y)=\max _{(s, t) \in B(x, y)} f(s, t)=\delta_{B}(f)(x, y), \\
& \lim _{P \rightarrow-\infty} K_{B}^{P}(f)(x, y)=\min _{(s, t) \in B(x, y)} f(s, t)=\varepsilon_{B}(f)(x, y),
\end{aligned}
$$

in which $\delta$ and $\varepsilon$ represent the texture features and edge pixels of the image, respectively, and the parallax field of the image boundary is $B(x, y)$. Therefore, the two-dimensional feature information of the image is represented as follows:

$$
P\left(y_{w_{3}} \mid x_{w_{3}}, \theta, \beta\right)=\frac{1}{Z\left(\beta_{i}\right)} P\left(y_{w_{3}} \mid x_{w_{3}}, \theta\right)\left(y_{w_{3}} \mid \beta_{1}\right)
$$

in which $Z\left(\beta_{1}\right)=\sum_{y_{w_{3}}} P\left(y_{w_{3}} \mid x_{w_{3}}, \theta\right)\left(y_{w_{3}} \mid \beta_{i}\right)$ is the image boundary overlapping the region. This paper adopts the image reconstruction method to obtain the Sanda motion 

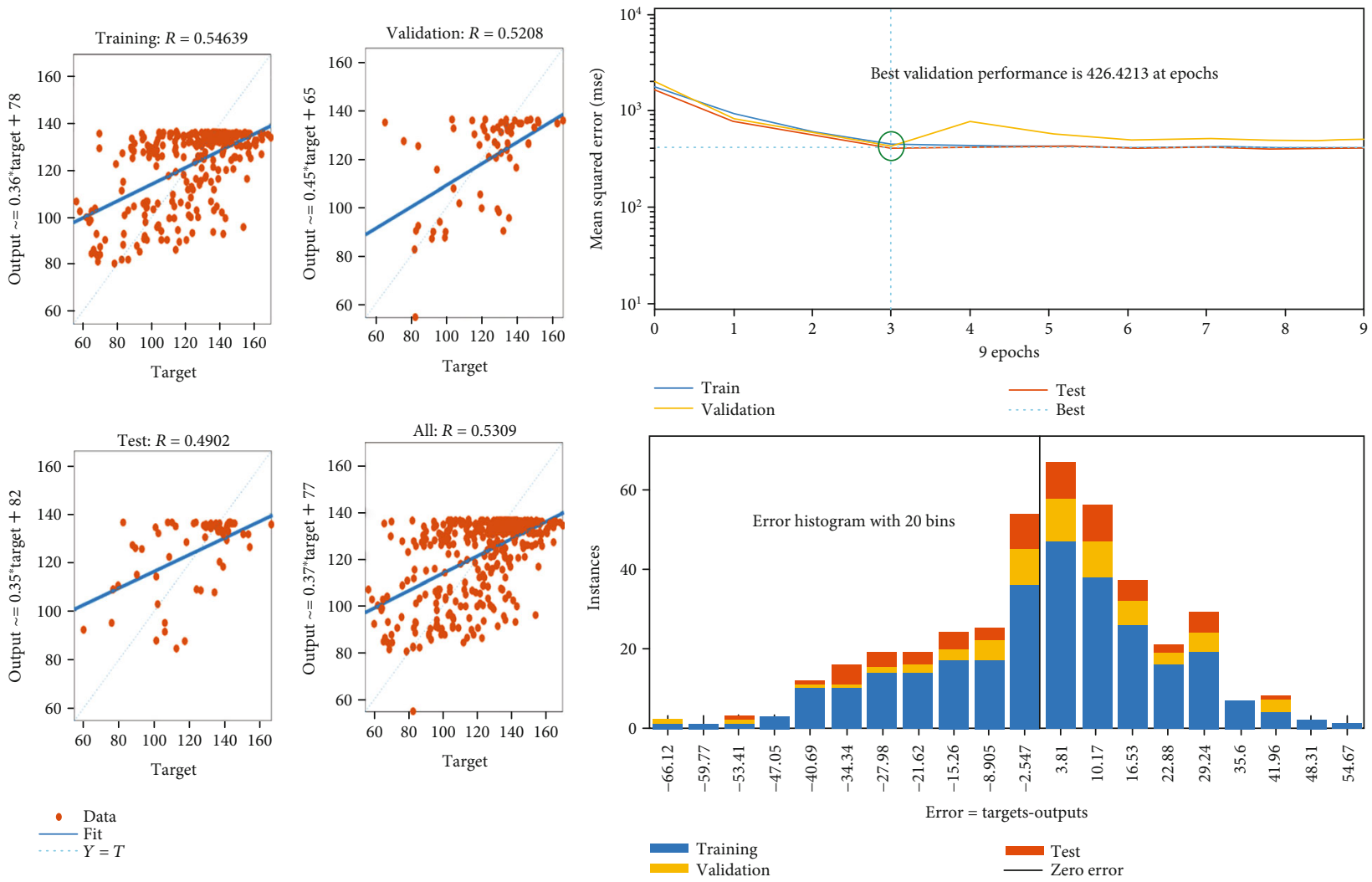

FIGURE 6: Compare training graphs with different methods.

reconstruction model based on the $3 \mathrm{D}$ image as shown in Figures 3 and 4 . It is clearly evident from Figures 3 and 4 that the feature extraction ability is conspicuous. The stronger the reconstruction ability of Sanda, the better the reconstruction effect.

\section{Analysis of Simulation Experiment}

The simulation experiment analysis of Sanda action 3D image reconstruction is carried out in the MATLAB simulation environment. The method of fixed-point frame scanning is used to collect the original image. The frequency of scanning is $f=12 \mathrm{kHz}, T_{m}=14$, the size of the scanning image is $2000 \times 2000$ pixels, and the image acquisition is carried out. The Gaussian noise of image quality is zero, and the variance is 0.24 . Besides the above simulation parameters, the $3 \mathrm{D}$ reconstruction quality is carried out. In order to compare the performance, three distinct methods are adopted for dimension reconstruction. The quality of the reconstruction of various methods is tested by distinct methods, and the comparison results are shown in Figure 5.

The network training results during the comparison of unequal methods are shown in Figure 6.

Figure 6 shows that the error of Sanda reconstruction using this method is lower than that of traditional methods. In contrast with conventional methods, the error is reduced by $12.5 \%$, and the SNR of the reconstructed output is increased by $23.4 \mathrm{~dB}$.

\section{Conclusions}

Aiming at improving the visual effect of Sanda 3D image reconstruction, a three-dimensional image reconstruction technique for Sanda action based on frame adjacent phase compensation and digital image stabilization is proposed. Digital frame scanning and corner detection are carried out to images. The adjacent phase compensation method is used to match feature points and segment gray pixels. By means of extracting the key feature points, the fast frame detection method is wielded to stabilize the image of the digital image so that the image quality of image reconstruction is ameliorated. The experimental results imply that this method is equipped with excellent image output effect as well as superior application value on guidance and optimization.

\section{Data Availability}

The data used to support the findings of this study are included in the article.

\section{Conflicts of Interest}

The author declares that they have no conflicts of interest.

\section{Acknowledgments}

This work was supported by the National Social Science Foundation of China (No.15XMZ063) and the Guangxi 
Higher Education Undergraduate Teaching Reform Project (No.2019JGA174).

\section{References}

[1] B. B. Amor, J. Su, and A. Srivastava, "Action recognition using rate-invariant analysis, of skeletal shape trajectories," IEEE Transactions on Pattern Analysis \& Machine Intelligence, vol. 11, no. 1, pp. 1-13, 2016.

[2] Y. Weiwu, A Framework and Modeling Method of Data-Driven Soft Sensors Based on Semisupervised Gaussian Regression, Industrial \& Engineering Chemistry Research, 2016.

[3] M. I. Jie, Z. Peng, and Y. U. Haipeng, "Large data clustering algorithm based on particle swarm differential perturbation optimization[J]," Journal of Henan University of Engineering (Natural Science Edition), vol. 28, no. 1, pp. 63-68, 2016.

[4] A. Aravind and M. Beena, "Electrochemical sensor based on nanostructured ion imprinted polymer for the sensing and extraction of $\mathrm{Cr}$ (III) ions from industrial wastewater," Polymer International, vol. 67, no. 12, pp. 1595-1604, 2018.

[5] D. Y. Jia and F. Z. Zhang, "A collaborative filtering recommendation algorithm based on double neighbor choosing strategy[J]," Journal of Computer Research and Development, vol. 50, no. 5, pp. 1076-1084, 2013.

[6] W. Chi, W. Chen, and Y. Wang, "Scalable influence maximization for independent cascade model in large-scale social networks[J]," Data Mining \& Knowledge Discovery, vol. 49, no. 3, pp. 498-504, 2011.

[7] J. X. Cao, D. Dong, S. Xu, X. Zheng, B. Liu, and J. Luo, “A kcore based algorithm for influence maximization in social networks[J]," Chinese Journal of Computers, vol. 38, no. 2, pp. 238-248, 2015.

[8] Y. Wang, F. Zhang, G. Fang, Y. Ji, S. Ye, and X. Zhang, "A novel ultrawideband exponentially tapered slot antenna of combined electric-magnetic type[J]," IEEE Antennas and Wireless Propagation Letters, vol. 15, pp. 1226-1229, 2016.

[9] G. Gennarelli and F. Soldovieri, "Multipath ghosts in radar imaging: physical insight and mitigation strategies," IEEE Journal of Selected Topics in Applied Earth Observations and Remote Sensing, vol. 8, no. 3, pp. 1078-1086, 2014.

[10] A. Hu, R. Zhang, and D. Yin, "Image quality assessment using a SVD-based structural projection," Signal Processing: Image Communication, vol. 29, no. 3, pp. 293-302, 2014.

[11] S. G. Li, Y. Jia, and Y. Guo, "Moving target tracking algorithm based on improved Camshaft for through-wall-radar imaging," Journal of Computer Applications, vol. 38, no. 2, pp. 528-532, 2018.

[12] Z. Moghaddam and M. Piccardi, "Training initialization of hidden Markov models in human action recognition," IEEE Transactions on Automation Science \& Engineering, vol. 11, no. 2, pp. 394-408, 2014.

[13] Z. K. Ma and W. H. Chang, "Friction torque calculation method of ball bearings based on rolling creepage theory," Journal of Mechanical Engineering, vol. 53, no. 22, pp. 219224, 2017.

[14] W. H. LI and H. Y. NI, "An improved AdaBoost training algorithm[J]," Journal of Jilin University (Science Edition), vol. 49, no. 3, pp. 498-504, 2011.

[15] L. G. Ramos, S. F. G. Vegas, and F. M. Martin, "Anisotropic diffusion filter with memory based on speckle statistics for ultrasound images," IEEE Transactions on Image Processing, vol. 24, no. 1, pp. 345-358, 2016.

[16] X. X. Shen, H. Zhang, and Z. Gao, "Behavior recognition algorithm based on Kinect and pyramid feature," Journal of Optoelectronics Laser, vol. 14, no. 2, pp. 357-363, 2014.

[17] G. H. Tian, J. Q. Yin, and X. Han, "A new method of human behavior recognition based on joint information," Robot, vol. 36, no. 3, pp. 285-292, 2014.

[18] Q. Y. Tan, H. Leung, and Y. Song, "Multipath ghost suppression for through-the-wall-radar," IEEE Transactions on Aerospace and Electronic Systems, vol. 50, no. 3, pp. 2284-2292, 2014.

[19] C. Xiu and F. Ba, "Target tracking based on the improved Cam shift method//CCDC 2016," in Proceedings of the 2016 Chinese Control and Decision Conference, pp. 3600-3604, Piscataway, NJ, 2016.

[20] Q. G. Zhang, F. Cai, and Z. Q. Li, "Human action recognition based on coupled multi-hidden Markov model and depth image data," Journal of Computer Applications, vol. 38, no. 2, pp. 454-457, 2018.

[21] H. Liang, J. Zou, K. Zuo, and K. Muhammad Junaid, “An improved genetic algorithm optimization fuzzy controller applied to the wellhead back pressure control system," Mechanical Systems and Signal Processing, vol. 142, p. 106708, 2020. 